\title{
Visualizations of direct fuel injection effects in a supersonic cavity flameholder
}

\author{
Hariswaran Sitaraman, ${ }^{*}$ Nicholas Brunhart-Lupo, Marc Henry de Frahan, Shashank Yellapantula, \\ Bruce Perry, Jon Rood $\odot$, Ray Grout, Marc Day, Roba Binyahib, and Kenny Gruchalla $\odot$ \\ National Renewable Energy Laboratory, 15013 Denver West Parkway, Golden, Colorado 80401, USA
}

(Received 6 August 2021; published 15 November 2021)

\begin{abstract}
This paper is associated with a video winner of a 2020 American Physical Society's Division of Fluid Dynamics (DFD) Gallery of Fluid Motion Award for work presented at the DFD Gallery of Fluid Motion. The original video is available online at the Gallery of Fluid Motion, https://doi.org/10.1103/APS.DFD.2020.GFM.V0026.
\end{abstract}

DOI: 10.1103/PhysRevFluids.6.110504

Supersonic combustion has received considerable interest in recent years due to emphasis on hypersonic vehicle development [1], reusable launch systems, and air-breathing rocket engines [2]. Combustion and flame stabilization at high supersonic flows is challenging mainly due to small residence times for fuel-oxidizer mixing and ignition. Cavity-based flameholders are a viable technique for providing flame stabilization in these applications. They enable flow deceleration and recirculation, thus increasing the residence time for adequate mixing of fuel and for subsequent combustion. Direct fuel injection into the cavity has been found to be advantageous compared to passive injection strategies with regard to greater control of local stoichiometry and fuel residence times [3]; however, a theoretical understanding of turbulent mixing and combustion between the fuel jet and supersonic air stream has not yet been fully developed. The main contribution of this work is to address these interactions via high-fidelity combustion simulations and cutting-edge visualization.

We employ PELEC [4,5], an open-source compressible reacting flow solver, to study the combustion and flow dynamics in a representative cavity flameholder. We solve the compressible multispecies Navier-Stokes equations with finite-rate $\mathrm{H}_{2}$-air chemistry. The solver employs adaptive-mesh-refinement (AMR) techniques on a Cartesian grid framework with a cut-cell-based formulation for representing complex geometries. Adaptive-mesh refinement is enabled at locations with high density gradients, vorticity, and temperature for resolving shock waves, shear-layer turbulence, and flames, respectively. We use an open-source cinematic package BLENDER [6] that provides physics-based volume rendering for visualizing the flow and combustion phenomena resolved by our simulations.

The geometry examined in this work is shown in Fig. 1(a) and involves supersonic flow over a cavity with a ramp-shaped trailing edge. The use of a ramp shape has been found to be advantageous compared to a rectangular shape with regard to reduction in drag and shear-layer oscillations [7]. We study direct fuel injection into this cavity at two different locations, labeled as cases C1 and C2. The first step was to run a nonreacting simulation without fuel injection to statistically stationary

\footnotetext{
*Hariswaran.Sitaraman@nrel.gov
}

Published by the American Physical Society under the terms of the Creative Commons Attribution 4.0 International license. Further distribution of this work must maintain attribution to the author(s) and the published article's title, journal citation, and DOI. 


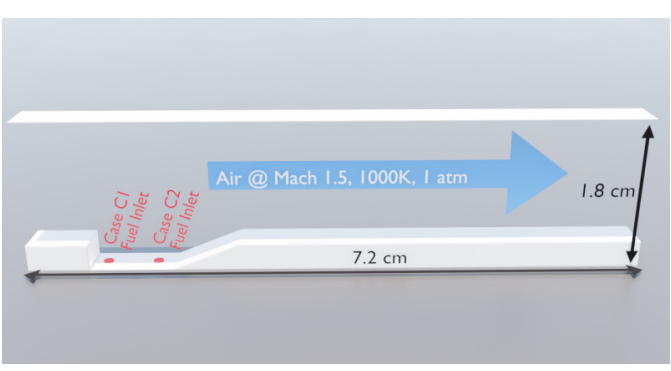

(a)
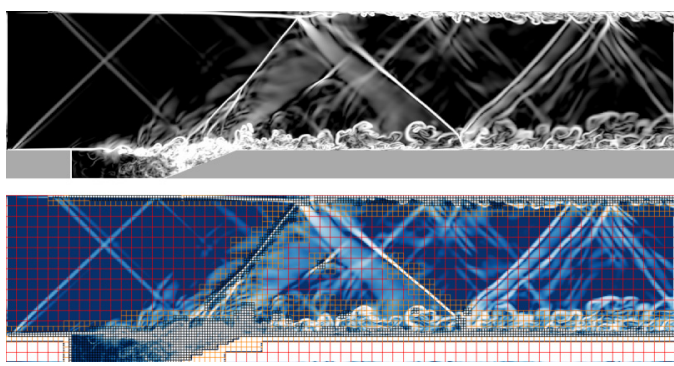

(c)

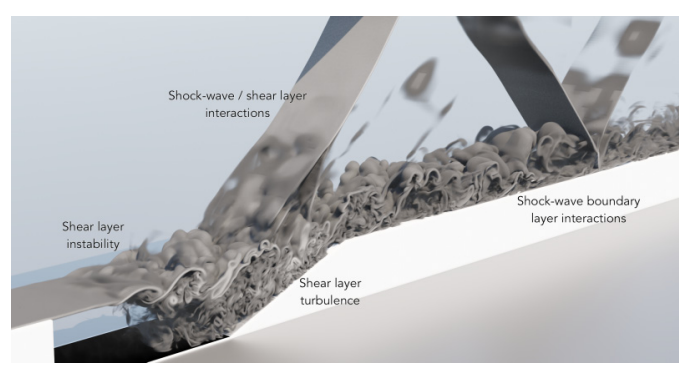

(b)

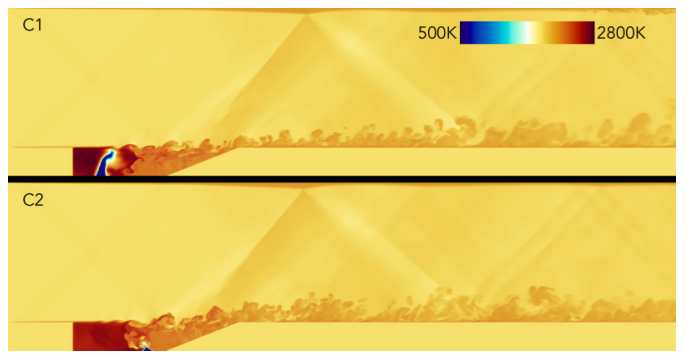

(d)

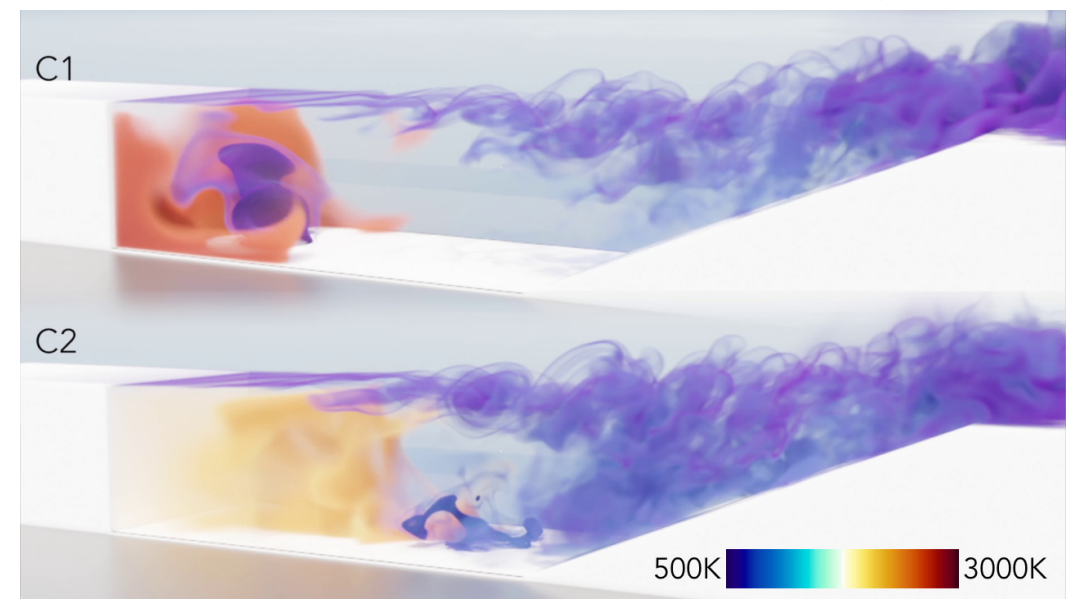

(e)

FIG. 1. (a) Schematic of the cavity geometry used in our simulation, (b) flow features from a nonreacting simulation such as shock waves and shear layers, (c) numerical schlieren and corresponding numerical grid with AMR where each box is an $8 \times 8 \times 8$ grid, (d) temperature snapshots on a midplane slice, and (e) temperature distribution within the cavity at statistical steady state for cases $\mathrm{C} 1$ and $\mathrm{C} 2$. Please refer to the full video at https://doi.org/10.1103/APS.DFD.2020.GFM.V0026.

state. The stabilized flow structures such as oblique shock waves, shear-layer turbulence, and shockboundary-layer interactions are as shown in Fig. 1(b). These flow structures are resolved using three levels of AMR (base level at $512 \times 128 \times 32$ grid), as indicated in Fig. 1(c), which shows a numerical schlieren snapshot along with the corresponding computational mesh.

A reacting flow simulation for each injection location was initialized using the nonreacting flow fields. Figure 1(d) shows a snapshot of temperature for cases $\mathrm{C} 1$ and $\mathrm{C} 2$ after achieving statistically stationary states in the reacting simulation. The compression waves emanating from 
the shear layer are only slightly altered by fuel injection, with greater impact in case $\mathrm{C} 1$ due to farther jet penetration. The important difference between the two injection scenarios, elucidated from the visualization, is the interaction between the fuel jet and shear layer as shown in Fig. 1(e). The fuel jet in case $\mathrm{C} 1$ is injected into a low-momentum region, while the downstream $\mathrm{C} 2$ injection is in the vicinity of shear-layer turbulence. There is greater penetration of the fuel jet into the cavity in case $\mathrm{C} 1$, while it disintegrates close to the inlet in case $\mathrm{C} 2$. The increased jet penetration into the cavity for case $\mathrm{C} 1$ results in a low-frequency oscillatory behavior $(\sim 4 \mathrm{kHz})$ between pressure and heat release [4], while high-frequency oscillations $(\sim 30 \mathrm{kHz})$ consistent with closed-box acoustics are observed in case $\mathrm{C} 2$. Furthermore, case $\mathrm{C} 1$ results in richer combustion and higher peak temperatures, while enhanced mixing in case $\mathrm{C} 2$ enables leaner combustion at lower temperatures. This high-fidelity simulation study, enabled by AMR, state-of-the-art visualization tools, and high-performance computing, elucidates important implications with regard to direct fuel injection, with downstream injection within the cavity potentially having more favorable properties when designing cavity flameholders.

We acknowledge funding from the U.S. Department of Energy's Exascale Computing Project (No. 17-SC-20-SC) and supercomputing resources at the National Renewable Energy Laboratory and the Oak Ridge Leadership Computing Facility. The U.S. Government retains and the publisher, by accepting the article for publication, acknowledges that the U.S. Government retains a nonexclusive, paid-up, irrevocable, worldwide license to publish or reproduce the published form of this work, or allow others to do so, for U.S. Government purposes.

[1] D. B. Le, C. P. Goyne, R. H. Krauss, and J. C. McDaniel, Experimental study of a dual-mode scramjet isolator, J. Propul. Power 24, 1050 (2008).

[2] J. Taylor, F. Flanagan, A. Dunlop, S. D. Grimshaw, and R. Miller, Super aggressive S-ducts for air breathing rocket engines, J. Turbomach. 143, 061015 (2021).

[3] F. W. Barnes and C. Segal, Cavity-based flameholding for chemically-reacting supersonic flows, Prog. Aerosp. Sci. 76, 24 (2015).

[4] H. Sitaraman, S. Yellapantula, M. T. H. de Frahan, B. Perry, J. Rood, R. Grout, and M. Day, Adaptive mesh based combustion simulations of direct fuel injection effects in a supersonic cavity flame-holder, Combust. Flame 232, 111531 (2021).

[5] https://github.com/AMReX-Combustion/PeleC.

[6] Blender online community, Blender-A 3D modelling and rendering package (Blender Foundation, Blender Institute, Amsterdam, 2021).

[7] X. Zhang, A. Rona, and J. A. Edwards, The effect of trailing edge geometry on cavity flow oscillation driven by a supersonic shear layer, Aeronaut. J. 102, 129 (1998). 